\title{
Implications of Initial LHC Searches for Supersymmetry
}

\author{
O. Buchmueller ${ }^{\mathrm{a}}$, R. Cavanaugh ${ }^{\mathrm{b}, \mathrm{c}}$, D. Colling ${ }^{\mathrm{a}}$, A. De Roeck ${ }^{\mathrm{d}, \mathrm{e}}$, M.J. Dolan ${ }^{\mathrm{f}}$, J.R. Ellis ${ }^{\mathrm{d}, \mathrm{g}}$,

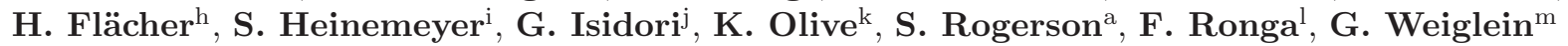 \\ ${ }^{a}$ High Energy Physics Group, Blackett Laboratory, Imperial College, Prince Consort Road, \\ London SW7 2AZ, UK \\ ${ }^{\mathrm{b}}$ Fermi National Accelerator Laboratory, P.O. Box 500, Batavia, Illinois 60510, USA

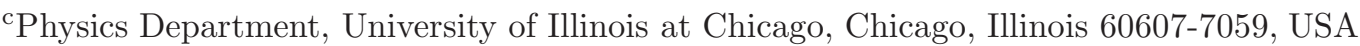 \\ ${ }^{\mathrm{d}} \mathrm{CERN}, \mathrm{CH}-1211$ Genève 23, Switzerland \\ eAntwerp University, B-2610 Wilrijk, Belgium \\ ${ }^{\mathrm{f}}$ Institute for Particle Physics Phenomenology, University of Durham, South Road, Durham DH1 3LE, UK \\ 'Theoretical Physics and Cosmology Group, Department of Physics, King's College London, London \\ WC2R 2LS, UK
}

${ }^{\mathrm{h}}$ Department of Physics and Astronomy, University of Rochester, Rochester, New York 14627, USA

instituto de Física de Cantabria (CSIC-UC), E-39005 Santander, Spain

jINFN, Laboratori Nazionali di Frascati, Via E. Fermi 40, I-00044 Frascati, Italy

${ }^{\mathrm{k}}$ William I. Fine Theoretical Physics Institute, University of Minnesota, Minneapolis, Minnesota 55455, USA

${ }^{1}$ Institute for Particle Physics, ETH Zürich, CH-8093 Zürich, Switzerland

mDESY, Notkestrasse 85, D-22607 Hamburg, Germany

The CMS and ATLAS Collaborations have recently published the results of initial direct LHC searches for supersymmetry analyzing $\sim 35 / \mathrm{pb}$ of data taken at $7 \mathrm{TeV}$ in the centre of mass. We incorporate these results into a frequentist analysis of the probable ranges of parameters of simple versions of the minimal supersymmetric extension of the Standard Model (MSSM), namely the constrained MSSM (CMSSM), a model with common nonuniversal Higgs masses (NUHM1), the very constrained MSSM (VCMSSM) and minimal supergravity (mSUGRA). We present updated predictions for the gluino mass, $m_{\tilde{g}}$, the light Higgs boson mass, $M_{h}, \operatorname{BR}\left(B_{s} \rightarrow \mu^{+} \mu^{-}\right)$and the spin-independent dark matter scattering cross section, $\sigma_{p}^{\mathrm{SI}}$. The CMS and ATLAS data make inroads into the CMSSM, NUHM1 and VCMSSM (but not mSUGRA) parameter spaces, thereby strengthening previous lower limits on sparticle masses and upper limits on $\sigma_{p}^{\text {SI }}$ in the CMSSM and VCMSSM. The favoured ranges of $\mathrm{BR}\left(B_{s} \rightarrow \mu^{+} \mu^{-}\right)$in the CMSSM, VCMSSM and mSUGRA are close to the Standard Model, but considerably larger values of $\operatorname{BR}\left(B_{s} \rightarrow \mu^{+} \mu^{-}\right)$are possible in the NUHM1. Applying the CMS and ATLAS constraints improves the consistency of the model predictions for $M_{h}$ with the LEP exclusion limits. 
The results of experiments at the LHC will be make-or-break for supersymmetry. Multiple analyses have shown that the ATLAS and CMS experiments at the LHC have excellent chances of discovering supersymmetry (SUSY) 112 if it provides the astrophysical cold dark matter [3], and/or if sparticles are light enough to render natural the electroweak mass scale [4, and/or SUSY explains the apparent discrepancy between experimental measurement and the Standard Model (SM) prediction for the anomalous magnetic moment of the muon, $(g-2)_{\mu}$ 56677. In parallel, the CDF, D $\varnothing$, ATLAS and CMS experiments should be able to establish or disprove the existence of a SM-like Higgs boson weighing less than about $135 \mathrm{GeV}$, which is the upper bound predicted by the minimal supersymmetric extension of the Standard Model (MSSM) 89, provided that SUSY is realized at the $\mathrm{TeV}$ scale.

In anticipation of the LHC start-up, many groups have ventured estimates of the possible masses of supersymmetric particles in variants of the MSSM 10. The unconstrained MSSM contains too many parameters for a full exploration of its parameter space to be possible using present data. Therefore, we have focused on making estimates within the constrained MSSM (CMSSM) 1112 in which soft SUSY-breaking mass parameters are assumed to be universal at the GUT scale, in the simplest generalization of this model in which the universality is relaxed to allow non-universal Higgs masses (NUHM1) 1314]15, in a very constrained model in which a supplementary relation is imposed on trilinear and bilinear soft SUSY-breaking masses (VCMSSM) 16, and in minimal supergravity (mSUGRA) in which, in addition, the gravitino mass $m_{3 / 2}$ is set equal to the common soft SUSY-breaking scalar mass $m_{0}$ before renormalization 16]17.

Our estimates [12]14]15/17/18] have been made in a frequentist approach, in which we construct a global likelihood function with contributions from precision electroweak observables, $B$-physics observables, $(g-2)_{\mu}$ and the astrophysical cold dark matter density $\Omega_{\chi} h^{2}$ as well as the limits from the direct searches for Higgs bosons and sparticles at LEP. Our best fits in the CMSSM,
NUHM1, VCMSSM and mSUGRA all suggested that sparticles should be relatively light, perhaps even within the reach of early runs of the LHC.

The first results of initial direct searches for SUSY in data recorded by the CMS and ATLAS detectors analyzing $\sim 35 / \mathrm{pb}$ of integrated luminosity of collisions at $7 \mathrm{TeV}$ in the centre of mass taken in 2010 have now been published [19/20. In this paper we combine these newly-published results with our previous analyses to give updated predictions for the preferred regions of parameter space, gluino and Higgs masses, $\operatorname{BR}\left(B_{s} \rightarrow \mu^{+} \mu^{-}\right)$ and the spin-independent dark matter scattering cross section $\sigma_{p}^{\text {SI }}$ in the CMSSM, NUHM1, VCMSSM and mSUGRA frameworks.

We recall that the CMSSM [11] has four input parameters: the universal soft SUSY-breaking scalar and gaugino masses $\left(m_{0}, m_{1 / 2}\right)$, a universal trilinear soft SUSY-breaking parameter $A_{0}$ and the ratio of Higgs v.e.v.'s $\tan \beta$, as well as the sign of $\mu$ (the magnitude of $\mu$ and the bilinear SUSY-breaking parameter $B_{0}$ are fixed by the electroweak vacuum conditions). Concerning the latter, the results on $(g-2)_{\mu}[5] 617$ strongly favor a positive sign. In the NUHM1 [13, a common soft SUSY-breaking contribution to the masses of the two Higgs doublets is allowed to vary independently, so there are five independent parameters. On the other hand, the VCMSSM [16] imposes the supplementary constraint $B_{0}=A_{0}-m_{0}$ on the CMSSM, thereby removing $\tan \beta$ as a free input and leaving three parameters, on which the further constraint $m_{3 / 2}=m_{0}$ in mSUGRA [21] imposes a severe restriction.

Our analysis has been performed using the MasterCode 12/14/15/17/18/22]. The model parameter spaces are sampled using a Markov Chain Monte Carlo (MCMC) technique similar to that used in our previous papers. Our MCMC samplings of the CMSSM and NUHM1 parameter spaces each comprise some 25,000,000 points, whereas those of the VCMSSM and mSUGRA include some 30,000,000 and 17,000,000 points, respectively. The constraints are also treated similarly to our previous analyses, the significant changes being an updated value of the top quark mass, $m_{t}^{\exp }=173.3 \pm 1.1 \mathrm{GeV} 23$ and the use of the new $e^{+} e^{-}$determination 
of the SM contribution to $(g-2)_{\mu}$ [7, as described in [17 1. The numerical evaluation within the MasterCode 1214 15171822, combines SoftSUSY 27, FeynHiggs 9 28 29 290, SuFla 3132, SuperIso 33.34, a code providing supersymmetric predictions for electroweak observables based on 35/36], MicrOMEGAs 37/38139] and DarkSUSY [041, making extensive use of the SUSY Les Houches Accord 4243 .

The MasterCode is designed in such a way that the constraints from new observables can be taken into account and incorporated quickly and easily into the global likelihood function as 'afterburners' (i.e., as add-ons to the global $\chi^{2}$ function), provided that the contribution to the likelihood function from the new observable is available. The new ingredients in this analysis are the contributions from the direct SUSY searches performed by CMS and ATLAS [1920, which are incorporated as just such 'afterburners', via the procedures described below.

Previous studies 12 had indicated that $35 / \mathrm{pb}$ at $7 \mathrm{TeV}$ would provide sufficient sensitivity to probe regions of the $\left(m_{0}, m_{1 / 2}\right)$ planes favoured previously at the $95 \%$ and $68 \%$ confidence levels (CL), close to the previous best-fit points in the NUHM1 and CMSSM, though not as far as the best-fit points in the VCMSSM and mSUGRA. Therefore, the possibility that the actual experimental exclusion would be less than the expected sensitivity could not be excluded. However, this amount of luminosity could not be expected to lead to a $5-\sigma$ discovery of anomalous missing-transverse-energy events beyond those expected in the SM, and the interpretation of any excess would be ambiguous, since one could not expect to be able to discriminate between SUSY and other potential explanations. On the

\footnotetext{
${ }^{1}$ We have not updated our evaluation of $R_{B \rightarrow \tau \nu}=$ $\mathrm{BR}(B \rightarrow \tau \nu) / \mathrm{BR}(B \rightarrow \tau \nu)_{\mathrm{SM}}=1.43 \pm 0.43$ [15, though higher values have been reported in 24|25. All results are compatible within the errors: the main differences in the central values are related to the values of $\left|V_{u b}\right|$ and $f_{B}$. We have checked that using the value $R_{B \rightarrow \tau \nu}=2.07 \pm 0.54$ given in 25], corresponding to less conservative treatments of $\left|V_{u b}\right|$ and $f_{B}$, would have negligible effects on our fits, except to increase $\chi^{2}$ globally by $\sim 4$. Also we note that a large non-SM contribution to $B \rightarrow \tau \nu$ is not supported by $K \rightarrow \mu \nu$ data 26 .
}

other hand, it was to be expected that even non-observation of SUSY with this event sample would make an important contribution to the global likelihood function and possibly alter significantly the results of the fits.

The CMS result [19] is based on a search for multijet $+\mathbb{E}_{T}$ events without accompanying leptons. The 13 events found in the signal region were compatible with the $\sim 10.5$ expected from SM backgrounds with a probability value of $30 \%$ The observed result allowed CMS to set a 95\% CL (i.e., $1.96 \sigma$ ) upper limit of 13.4 signal events. This would correspond to $2.5 \pm(13.4-2.5) / 1.96=$ $2.5 \pm 5.6$ events for any possible signal, yielding $\chi_{\infty, \mathrm{CMS}}^{2}=0.85$ for large sparticle masses. The central CMS result, shown in Fig. 5 of 19 , is a $95 \% \mathrm{CL}$ exclusion contour in the $\left(m_{0}, m_{1 / 2}\right)$ plane of the CMSSM for the particular values $\tan \beta=3, A_{0}=0$ and $\mu>0$. However, the sensitivity of a search for multijet $+\mathbb{E}_{T}$ events is largely independent of these additional parameters within the CMSSM [19, and can also be taken over to the NUHM1, VCMSSM and mSUGRA models, which have similar signatures in these search channels.

Fig. 5 of [19] also presents a $\left(m_{0}, m_{1 / 2}\right)$ contour for the $95 \%$ CL exclusion expected in the absence of any signal, corresponding to $5.56 \times 1.96=10.9$ events. This contour would correspond to an apparent significance of $(10.9-2.5) / 5.56 \sim 1.5 \sigma$ and hence $\Delta \chi^{2} \sim 4$. The observed $95 \% \mathrm{CL}$ contour, on the other hand, corresponds to $\Delta \chi^{2}=5.99$. We approximate the impact of the new CMS constraint by $\Delta \chi_{\mathrm{CMS}}^{2} \sim \chi_{\infty, \mathrm{CMS}}^{2}\left|\left(M_{C} / M\right)-1\right|^{-p_{C}}$ (where $M \equiv \sqrt{m_{0}^{2}+m_{1 / 2}^{2}}$ ) for each ray in the $\left(m_{0}, m_{1 / 2}\right)$ plane, fitting the parameters $M_{C}, p_{C}$ by requiring $\Delta \chi^{2} \sim 4,5.99$ on the expected and observed $95 \%$ exclusion contours shown in Fig. 5 of 19$] 2$.

The ATLAS result [20] is based on a search for multijet $+\mathbb{E}_{T}$ events with one accompanying electron or muon. The 2 events found in the signal

\footnotetext{
${ }^{2}$ We have checked that this procedure gives a value for $\Delta \chi^{2}$ at the LM1 point in Fig. 5 of [19] that is consistent with the 19.2 events expected. We note that the functional form adopted for $\Delta \chi_{\mathrm{CMS}}^{2}$ is approximate, and our later estimates of systematic errors in our results are based on studies of alternative forms.
} 
region were compatible with the $\sim 4.1$ expected from SM backgrounds with a probability value of 16\%. The central ATLAS result, shown in Fig. 2 of 20, is again a $95 \%$ CL exclusion contour in the $\left(m_{0}, m_{1 / 2}\right)$ plane of the CMSSM for the particular values $\tan \beta=3, A_{0}=0$ and $\mu>0$, which is also only moderately dependent on these additional parameters within the CMSSM [20, and can also be taken over to the NUHM1, VCMSSM and mSUGRA models 3 . The observed result allowed ATLAS to set a 95\% CL (i.e., $1.96 \sigma$ ) upper limit on sparticle production that corresponds to 4.8 signal events and $\chi^{2}=5.99$. This would correspond to a downward fluctuation of $-2.1 \pm(4.8+2.1) / 1.96=-2.1 \pm 3.5$ events for any possible signal, yielding an estimate of $\chi_{\infty, \text { ATLAS }}^{2}=1.2$ for large sparticle masses.

In order to estimate the ATLAS sensitivity to sparticle masses in the parameter region beyond the observed 95\% CL, we follow [44, based on [45], in which the integrated luminosities $\mathcal{L}$ required for discovering sparticles of various masses were estimated. There it was found empirically that $\mathcal{M} \propto \mathcal{L}^{1 / 4}$, where $\mathcal{M}$ denotes a (similar) squark and gluino mass. This suggests that for equal squark and gluino masses the effective event rate $\propto \mathcal{M}^{-4}$. Using this as a guide, we assume that the effective numbers of events expected for relevant points in the $\left(m_{0}, m_{1 / 2}\right)$ plane scale as $\mathcal{M}^{-4}$. We then calculate the corresponding significances assuming the observed signal of $-2.1 \pm 3.5$ estimated above, to estimate the corresponding values of $\chi^{2}$. As an approximate analytic interpolating form, we use $\Delta \chi_{\text {ATLAS }}^{2} \sim \chi_{\infty, \text { ATLAS }}^{2}+\left(M_{A} / M\right)^{-p_{A}}$, fitting $M_{A}$ and $p_{A}$ along rays in the $\left(m_{0}, m_{1 / 2}\right)$ plane. Our analysis is based exclusively on published material 19204445, and we consider it prudent to assign a larger systematic error to our implementation of the ATLAS constraint.

As shown in Fig. 5 of [19] and Fig. 2 of [20, the direct physics reaches of the CMS and ATLAS data in the models studied are much greater than those of the earlier CDF, DØ and LEP2 searches for sparticles, so the latter do not make signif-

\footnotetext{
${ }^{3}$ This would not be the case for multi-lepton + jets + $E_{T}$ searches, which have a stronger dependence on $\tan \beta$, leading in particular to weaker bounds for large $\tan \beta$.
}

icant contributions to the global $\chi^{2}$ function in the regions of interest for our analysis. On the other hand, the LEP2 Higgs search [46|47] had an indirect reach in the $\left(m_{0}, m_{1 / 2}\right)$ plane that is comparable to those of the direct CMS and ATLAS constraints in the models studied, as we see explicitly later.

Conservatively, we do not attempt to combine the CMS and ATLAS constraints in the following discussion, which would require detailed modelling of their likelihood functions and a better understanding of correlations between observables in the CMS and ATLAS searches. The $\chi^{2}$ functions obtained by combining the CMS and ATLAS constraints separately with previous constraints in the $\left(m_{0}, m_{1 / 2}\right)$ planes for the CMSSM, NUHM1, VCMSSM and mSUGRA models are shown in Fig. 1. In each panel, the new $68 \%$ and 95\% CL contours incorporating the CMS (ATLAS) results are shown as red and blue dashed (solid) lines, and the corresponding previous contours are shown as dotted lines. (It should be noted that the updated values of $m_{t}$ and $(g-2)_{\mu}$ and some further technical improvements have changed slightly the best fits and likelihood contours with respect to our earlier publications 14 15 17.) We also indicate as open and full green stars the new best-fit points including the CMS and ATLAS results, respectively, and indicating the pre-LHC best-fit points by green 'snowflakes' 4. In panels where only one green star is visible, the CMS and ATLAS bestfit points lie on top of each other, within the $\sim 10 \mathrm{GeV}$ accuracy adopted for our numbers. The jagged boundaries of the 95\% CL CMSSM and NUHM1 contours at large $m_{0}$ and $m_{1 / 2}$ reflect the uncertainties in sampling the slow variations in their likelihood functions.

In Table1 we compare the best-fit points found in this paper incorporating the CMS and ATLAS constraints with pre-LHC results 14 15]17] in the CMSSM, NUHM1 and VCMSSM (the fits in mSUGRA are essentially unchanged when LHC

\footnotetext{
${ }^{4}$ We recall that in $\mathrm{mS} U G R A$ there is a secondary minimum of the likelihood function with $\Delta \chi^{2} \sim 4$ that is located at $\left(m_{0}, m_{1 / 2}\right) \sim(2110,130) \mathrm{GeV}$ [17, which is not visible in Fig. 1 and lies well beyond the initial LHC reach.
} 

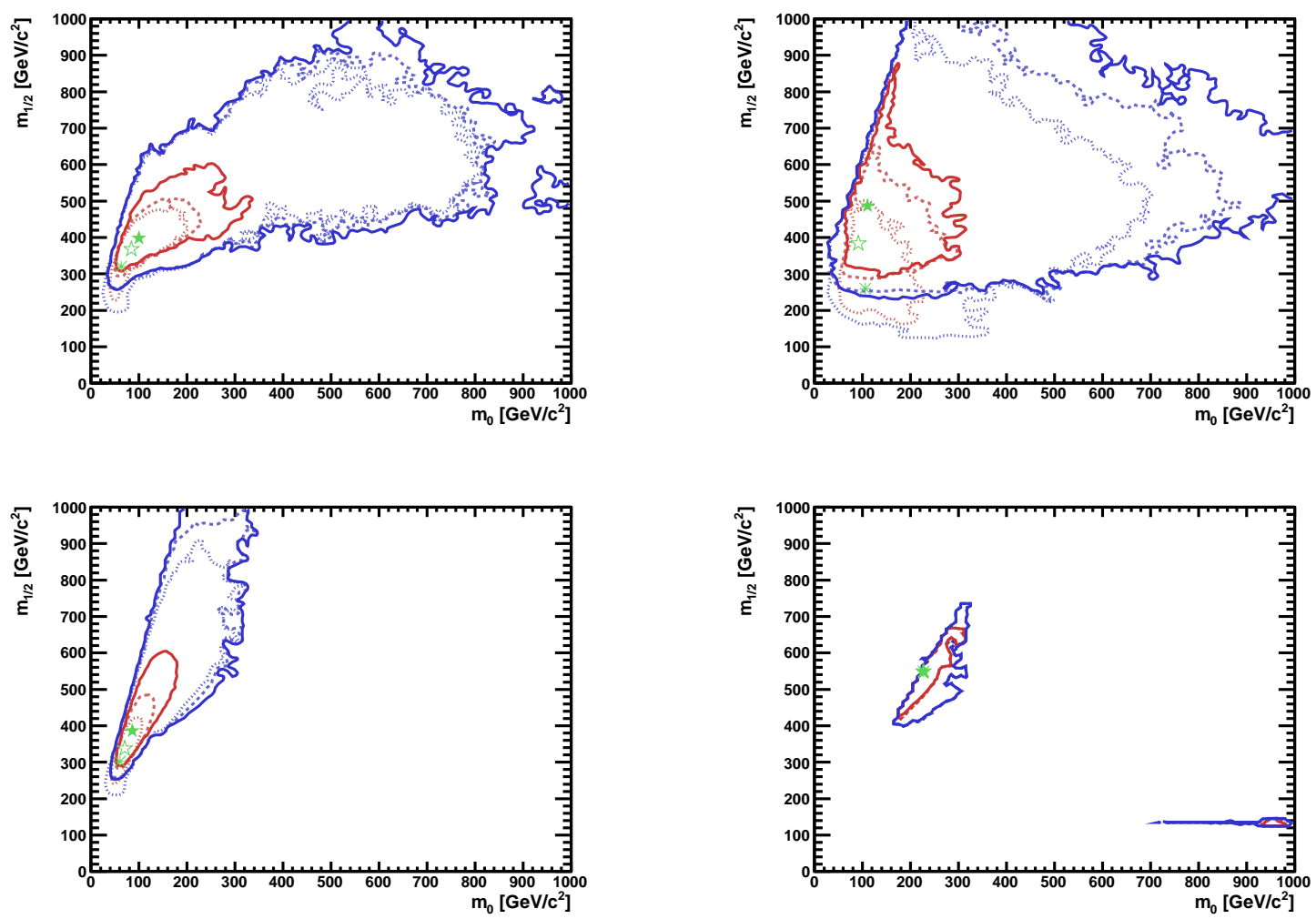

Figure 1. The $\left(m_{0}, m_{1 / 2}\right)$ planes in the CMSSM (upper left), NUHM1 (top right), VCMSSM (lower left) and mSUGRA (lower right). In each panel, we show the 68 and 95\% CL contours (red and blue, respectively) both after applying the CMS [19] and ATLAS [20] constraints (dashed and solid lines, respectively) and beforehand (dotted lines). Also shown as open (solid) green stars are the best-fit points found after applying the CMS (ATLAS) constraints in each model (see text), and as green 'snowflakes' the previous best-fit points.

data are included in the fits, only the best coannihilation fit is reported). In addition to the minimum value of $\chi^{2}$ and the fit probability in each scenario, we include the values of $m_{1 / 2}, m_{0}, A_{0}$ and $\tan \beta$ at all the best-fit points. We estimate systematic errors of $\sim 10 \%$ in the values for $m_{1 / 2}$ quoted in Table 1 for the best-fit points in the CMS analyses of the CMSSM, NUHM1 and VCMSSM, associated with the ambiguities in the implementations of the LHC constraints and the slow variations in the $\chi^{2}$ functions. In the cases of the ATLAS analyses, we have an additional systematic uncertainty in the implementation of the constraint, and estimate a somewhat larger error $\sim 20 \%$. Table 1 also shows the values of $M_{h}$ that would be estimated in each model if the LEP Higgs constraint were neglected.

The absences of supersymmetric signals in the CMS and ATLAS data $19 \mid 20$ invalidate portions of the CMSSM, NUHM1 and VCMSSM parameter spaces at low $m_{1 / 2}$ that were previously allowed at the $95 \%$ and $68 \%$ CLs 5, but do not im-

\footnotetext{
${ }^{5}$ Strictly speaking, the $\Delta \chi^{2}=5.99$ and 2.30 contours that we plot may not always correspond exactly to these CLs, but we ignore any possible differences here since previous studies 14/15/17] showed acceptable coverage,
} 


\begin{tabular}{|c||c|c|c|c|c|c||c|}
\hline Model & Minimum $\chi^{2}$ & Probability & $\begin{array}{c}m_{1 / 2} \\
(\mathrm{GeV})\end{array}$ & $\begin{array}{c}m_{0} \\
(\mathrm{GeV})\end{array}$ & $\begin{array}{c}A_{0} \\
(\mathrm{GeV})\end{array}$ & $\begin{array}{c}\tan \beta \\
M_{h} \text { (no LEP) } \\
(\mathrm{GeV})\end{array}$ \\
\hline \hline CMSSM & $(21.3)$ & $(32 \%)$ & $(320)$ & $(60)$ & $(-170)$ & $(11)$ & $(107.9)$ \\
with CMS & 22.0 & $29 \%$ & 370 & 80 & -340 & 14 & 112.6 \\
with ATLAS & 24.9 & $16 \%$ & 400 & 100 & -430 & 16 & 112.8 \\
\hline NUHM1 & $(19.3)$ & $(31 \%)$ & $(260)$ & $(110)$ & $(1010)$ & $(8)$ & $(121.9)$ \\
with CMS & 20.9 & $28 \%$ & 380 & 90 & 70 & 14 & 113.5 \\
with ATLAS & 23.3 & $18 \%$ & 490 & 110 & -630 & 25 & 116.5 \\
\hline VCMSSM & $(22.5)$ & $(31 \%)$ & $(300)$ & $(60)$ & $(30)$ & $(9)$ & $(109.3)$ \\
with CMS & 23.8 & $25 \%$ & 340 & 70 & 50 & 9 & 115.5 \\
with ATLAS & 27.1 & $13 \%$ & 390 & 90 & 70 & 11 & 117.0 \\
\hline mSUGRA & $(29.4)$ & $(6.1 \%)$ & $(550)$ & $(230)$ & $(430)$ & $(28)$ & $(107.8)$ \\
with CMS & 29.4 & $6.1 \%$ & 550 & 230 & 430 & 28 & 121.2 \\
with ATLAS & 30.9 & $5.7 \%$ & 550 & 230 & 430 & 28 & 121.2 \\
\hline
\end{tabular}

Table 1

Comparison of the best-fit points found previously in the CMSSM, the NUHM1, the VCMSSM and the coannihilation region of mSUGRA when the LHC constraints were not included (in parentheses) 14 15, 177, and the results of this paper incorporating the CMS [19] and ATLAS [20] constraints. In addition to the minimum value of $\chi^{2}$ and the fit probability in each scenario, we include the values of $m_{1 / 2}, m_{0}, A_{0}$ and $\tan \beta$ at all the best-fit points, as well as the predictions for $M_{h}$ neglecting the LEP constraint.

pinge significantly on the corresponding regions for mSUGRA. In the cases of the CMSSM and VCMSSM, the LHC data disfavour the low- $m_{1 / 2}$ tips of the coannihilation regions and increase significantly the best-fit values of $m_{1 / 2}$, as seen in Fig. 1 and Table 1. However, it should be kept in mind that $\chi^{2}$ is quite a shallow function of $m_{1 / 2}$ near the best-fit points, particularly in the NUHM1. In the case of the NUHM1, the CMS and ATLAS data disfavour a slice of parameter space at low $m_{1 / 2}$ and $m_{0}<400 \mathrm{GeV}$ extending from the coannihilation region towards the light-Higgs funnel discussed in 17. The CMS and particularly ATLAS data extend the 95\% CL regions to larger $m_{0}$ and $m_{1 / 2}$, particularly in the NUHM1.

While the CMS constraint, as seen in Table 1 . leads only to small increases in the global $\chi^{2}$ of $\sim 1$ for each model, the inclusion of the ATLAS

which should not have deteriorated in the presence of additional constraints. For recent studies in a related context, see [4]. constraint results in increases in $\chi^{2}$ by $\lesssim 4.6$. This indicates that there is no tension between the CMS data and previous constraints, whereas some tension may arise from the ATLAS constraint. Correspondingly, the fit probabilities in the different models are reduced by including the CMS and ATLAS constraints, but generally not to unacceptable levels. It should be recalled, though, that our ATLAS implementation is more uncertain, and our implementations of the constraints should, conservatively, each be assigned an uncertainty at least as large as the value of $\chi_{\infty}^{2}=0.85,1.2$ for CMS and ATLAS, respectively. On the other hand, the absence of a supersymmetric signal at the LHC with a luminosity of $\geq 1$ /fb at a centre-of-mass energy $\geq 7 \mathrm{TeV}$ would increase the global minimum of $\chi^{2}$ sufficiently to put severe pressure on these models.

Fig. 2 displays the effects of the CMS and ATLAS constraints on the $\left(\tan \beta, m_{1 / 2}\right)$ planes in the CMSSM, NUHM1, VCMSSM and mSUGRA 6.

${ }^{6}$ These planes are truncated at $\tan \beta=50$, so that per- 
We see that the ranges of $\tan \beta$ preferred at the $68 \% \mathrm{CL}$ are extended to significantly larger values in the CMSSM and (particularly) the NUHM1, to a lesser extent in the VCMSSM, and not at all in mSUGRA. The best-fit values of $\tan \beta$ are also increased in the CMSSM and NUHM1, but not significantly in the VCMSSM and mSUGRA. The increases are mainly due to the $(g-2)_{\mu}$ constraint: larger sparticle masses lead to a smaller supersymmetric contribution that can be compensated by a larger value of $\tan \beta$.

In the regions of parameter space of interest to the CMSSM, NUHM1, VCMSSM and mSUGRA, the direct reach of the LHC for supersymmetry is strongly influenced by the gluino mass, $m_{\tilde{g}}$. Accordingly, we display in Fig. 3 the one-parameter $\chi^{2}$ functions for $m_{\tilde{g}}$ relative to the minima in all these models. In each case, we display the new likelihood functions incorporating CMS and ATLAS data as dashed and solid lines, respectively, and those given by the pre-LHC fits as dotted lines. The plots display the $\Delta \chi^{2}$ contributions in each model relative to the best-fit points in that model. In this and subsequent figures, the oneparameter $\chi^{2}$ functions for mSUGRA are essentially unchanged when the LHC data are included but are shown for comparison purposes.

For each of the CMSSM, NUHM1 and VCMSSM, we see that the side of the likelihood function below the best-fit point is shifted to larger $m_{\tilde{g}}$ by similar amounts $\delta m_{\tilde{g}} \sim 100$ to $400 \mathrm{GeV}$. The best-fit values of $m_{\tilde{g}}$ in the CMSSM, NUHM1 and VCMSSM are now $\sim 800$ to $1000 \mathrm{GeV}$, and the sides of the likelihood function beyond the best-fit points rise quite similarly in these models, though more slowly in the NUHM1. In mSUGRA the most likely values of $m_{\tilde{g}}$ are unchanged by either CMS or ATLAS, lying in the range $\sim 1100$ to $\sim 1400 \mathrm{GeV}$, with a secondary minimum in the light Higgs funnel region at $m_{\tilde{g}} \sim 400 \mathrm{GeV}$ and large $m_{0}$ [17] that lies beyond the present reach of the LHC7.

The experimental search for a SM-like Higgs boson is heating up, with interesting prospects for both the Tevatron collider [49] and the LHC [12],

turbative RGE calculations remain reliable.

${ }^{7} \mathrm{~A}$ vestige of this region is visible in the VCMSSM at $\Delta \chi^{2} \sim 7.5$, see also the lower left panel of Fig. 2 see in particular 50. Accordingly, we display in Fig. 4 the one-parameter $\chi^{2}$ functions for the lightest MSSM Higgs mass $M_{h}$ in the CMSSM, NUHM1, VCMSSM and mSUGRA. In this figure we do not include the direct limits from LEP [46]4] or the Tevatron, so as to illustrate whether there is a conflict between these limits and the predictions of supersymmetric models. For each model we display the new likelihood functions incorporating the ATLAS data as solid lines, indicating the theoretical uncertainty in the calculation of $M_{h}$ of $\sim 1.5 \mathrm{GeV}$ by red bands. We also show, as dashed lines without red bands, the central value of the prediction based on the CMS constraint, and as dotted lines without red bands the pre-CMS predictions for $M_{h}$ (all discarding the LEP constraint).

In the case of the CMSSM, we see that the CMS and ATLAS constraints increase the consistency of the model prediction with the direct LEP limit on $M_{h}$, indicated by the yellow region: the best-fit value is found at $112.6(112.8) \mathrm{GeV}$ after the inclusion of the CMS (ATLAS) constraints (but still neglecting the LEP Higgs searches, see also Table 10, with an estimated theoretical error of $1.5 \mathrm{GeV}$. In the case of the NUHM1, apart from somewhat lower best-fit values of $M_{h}=$ 113.5 (116.5) GeV after including the CMS (ATLAS) constraint (we recall that the $\chi^{2}$ function was very shallow in the direction of lower masses), we see that the main effect of the LHC data is to increase substantially the one-parameter $\chi^{2}$ function at low masses $M_{h}<110 \mathrm{GeV}$. (It should be remembered that in the NUHM1 the LEP constraint is weakened at low $M_{h}$ because the $h Z Z$ coupling may be reduced, which is not possible in the CMSSSM, VCMSSM and mSUGRA [51/52. The LHC data render a large reduction less likely.) Now most of the preferred $M_{h}$ region in the NUHM1 is indeed above $\sim 114 \mathrm{GeV}$. In the case of the VCMSSM, the LHC constraint strongly disfavours a Higgs boson below the LEP limit. In the case of the mSUGRA coannihilation region, the global minimum of the pre-LHC $\chi^{2}$ with the LEP constraint disregarded was found in an isolated region at low $\left(m_{0}, m_{1 / 2}\right)$, resulting in $M_{h} \sim 108 \mathrm{GeV}$. The inclusion of either the CMS or ATLAS data removes this iso- 

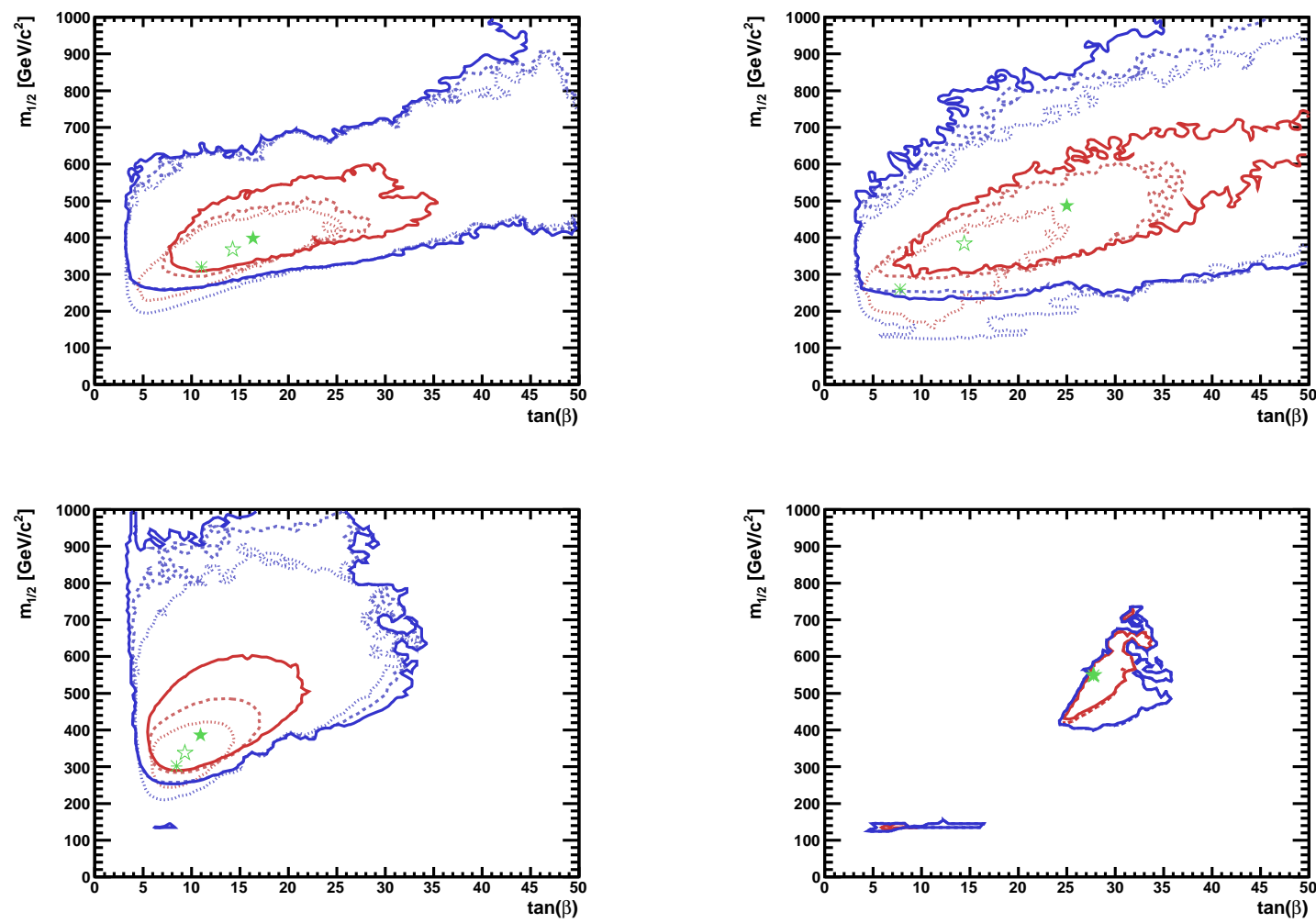

Figure 2. The $\left(\tan \beta, m_{1 / 2}\right)$ planes in the CMSSM (upper left), NUHM1 (top right), VCMSSM (lower left) and mSUGRA (lower right). The line styles and stars have the same meanings as in Fig. 1.

lated region, pushing the preferred range to larger $M_{h} \sim 121 \mathrm{GeV}$ compatible with the LEP constraint [46[47]. The global minimum with either the CMS or ATLAS constraint included is very similar to the pre-CMS fit with the LEP constraint included. In general, these plots support the remark made earlier that the LHC data have an effect comparable to the LEP $M_{h}$ limit in constraining the models studied.

For this reason, the LHC data do not have a great impact on the amounts of fine-tuning of parameters required. We have evaluated the finetuning measure proposed in 53] for the best pre(post-)LHC fits, finding in the CMSSM 100 (120) [140] before the LHC (with the CMS constraint) [with the ATLAS constraint]. The corresponding numbers in the other models are: NUHM 250
(230) [310], VCMSSM 130 (110) [140], mSUGRA 250 in all cases. 8

One of the other observables of potential interest for testing variants of the $\mathrm{MSSM}$ is $\operatorname{BR}\left(B_{s} \rightarrow\right.$ $\left.\mu^{+} \mu^{-}\right)$, and we recall that the sensitivity of the $\mathrm{LHCb}$ experiment from the data recorded during the 2010 LHC run approaches that already achieved by the CDF and D $\varnothing$ experiments [55]. As seen in Fig. 5. we find that the effects of the LHC data on this observable are negligible in mSUGRA and very small in the VCMSSM. However, the larger values of $\tan \beta$ now favoured in the CMSSM increase the likelihood of an enhancement in $\operatorname{BR}\left(B_{s} \rightarrow \mu^{+} \mu^{-}\right)$beyond the SM value. As was discussed previously [17], the best

${ }^{8}$ See 54 for a contrasting view that does not take into account the LEP constraint on $M_{h}$. 

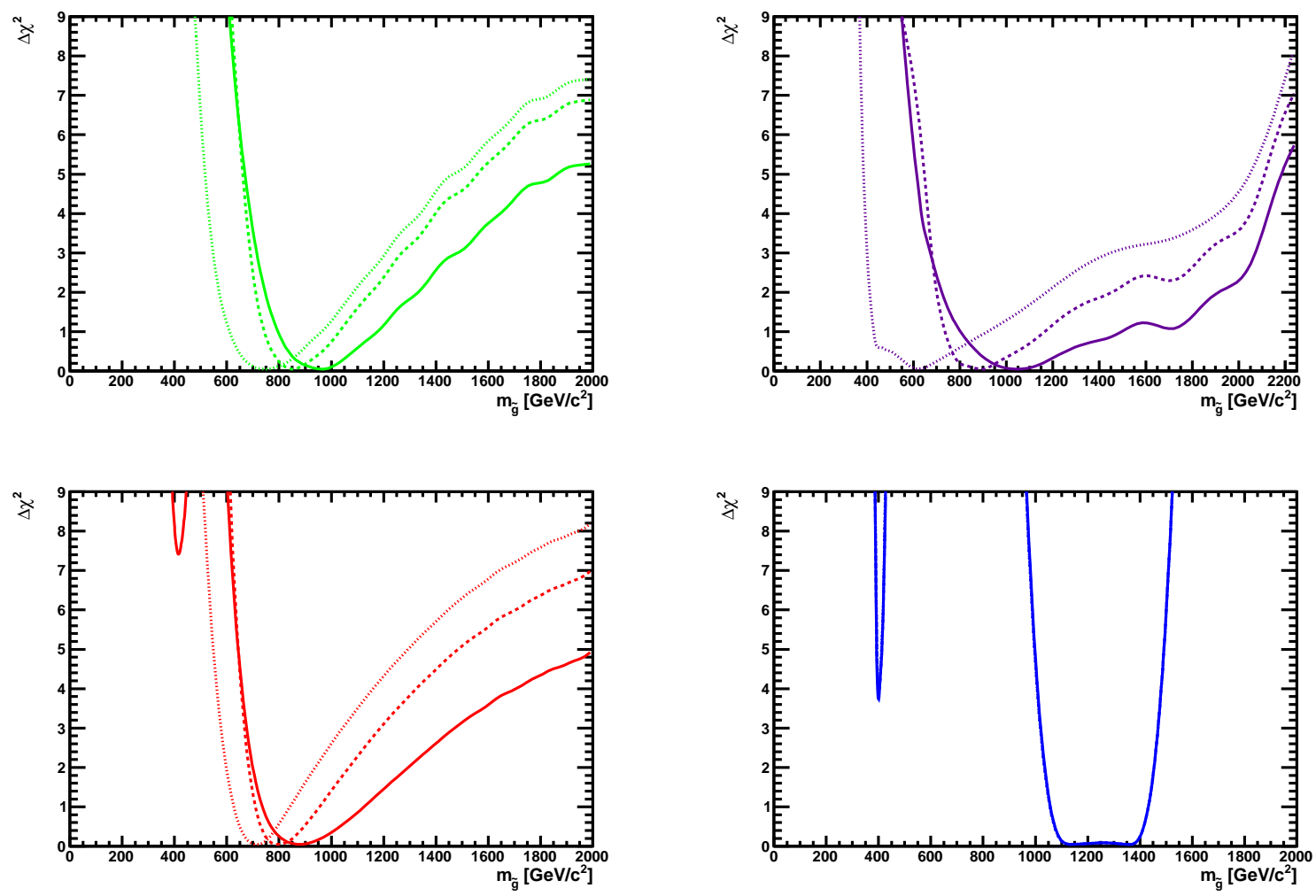

Figure 3. The one-parameter $\chi^{2}$ likelihood functions for the gluino mass $m_{\tilde{g}}$ in the CMSSM (upper left), NUHM1 (top right), VCMSSM (lower left) and mSUGRA (lower right). In each panel, we show the $\chi^{2}$ function including the CMS and ATLAS [1920] constraints as dashed and solid lines, respectively, and the previous $\chi^{2}$ function as a dotted line.

prospects for a deviation from the SM prediction for $\operatorname{BR}\left(B_{s} \rightarrow \mu^{+} \mu^{-}\right)$are in the NUHM1, where the best-fit value is significantly larger than in the SM and substantially larger values are now possible, thanks to the larger values of $\tan \beta$ now preferred. On the other hand, the best-fit values for $\operatorname{BR}\left(B_{s} \rightarrow \mu^{+} \mu^{-}\right)$in the CMSSM, VCMSSM and mSUGRA are peaked within $10 \%$ of the SM value, indistinguishable from the SM given the theoretical and experimental uncertainties.

Finally, we recall that it is expected that the sensitivities of direct searches for the spinindependent scattering of dark matter particles on heavy nuclei will soon be increasing substantially [56]. Accordingly, we display in
Fig. [6] the one-parameter $\chi^{2}$ functions for the spin-independent neutralino dark matter scattering cross section $\sigma_{p}^{\text {SI }}$ in the CMSSM, NUHM1, VCMSSM and mSUGRA. We display the new likelihood functions incorporating CMS and ATLAS data and calculated assuming the default value $\Sigma_{\pi N}=64 \mathrm{MeV}$ [57] as dashed and solid lines, as previously, and those given by the previous fits as dotted lines. We see that in the CMSSM and VCMSSM the LHC constraint reduces substantially the likelihood of relatively large values of $\sigma_{p}^{\text {SI }}>10^{-44} \mathrm{~cm}^{2}$ for $\Sigma_{\pi N}=$ $64 \mathrm{MeV}$, whereas the likelihood functions are little changed in mSUGRA and the NUHM1, due in the latter case to compensation between pref- 

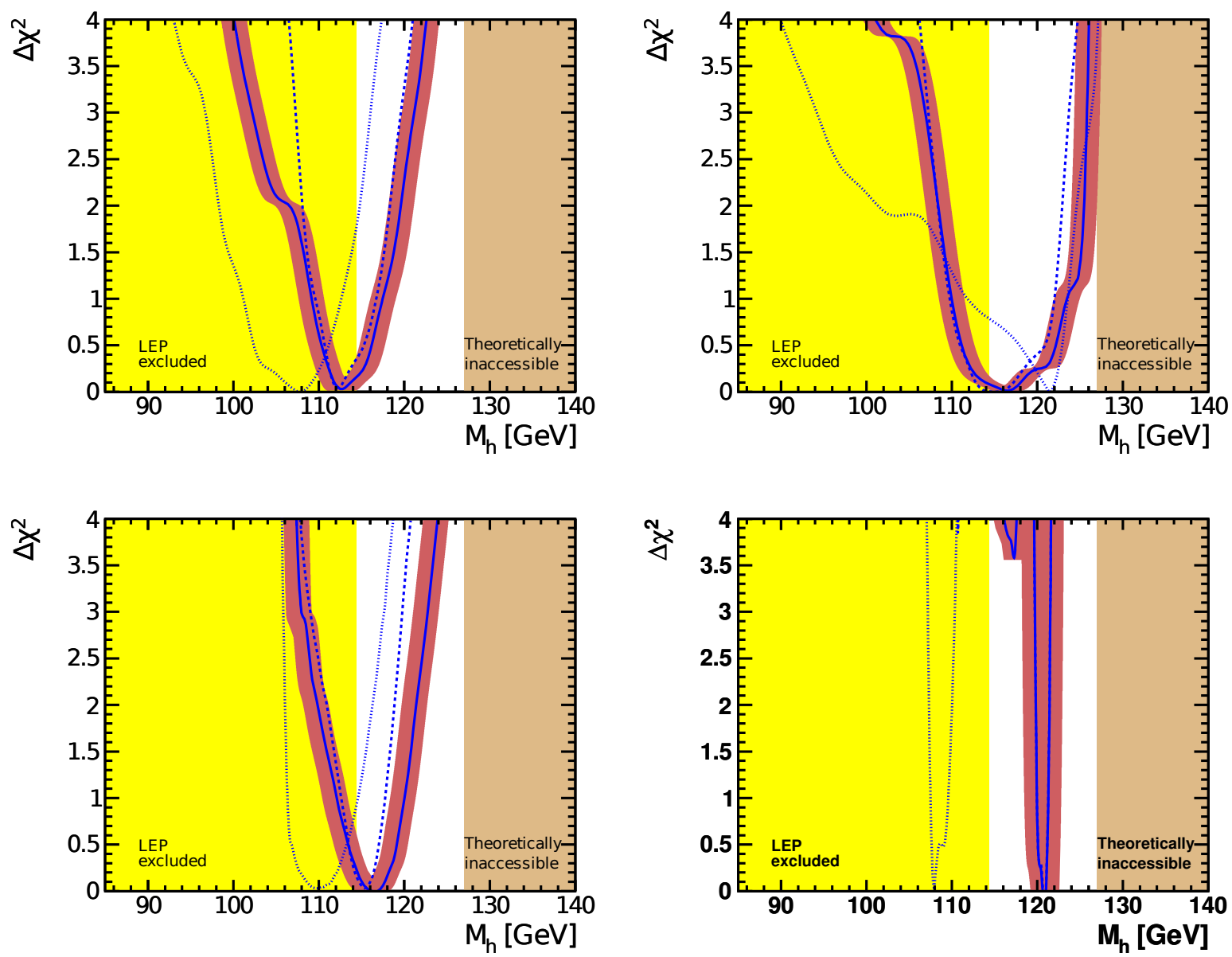

Figure 4. The one-parameter $\chi^{2}$ likelihood functions for the lightest MSSM Higgs mass $M_{h}$ in the CMSSM (upper left), NUHM1 (top right), VCMSSM (lower left) and mSUGRA (lower right). In each panel, we show the $\chi^{2}$ functions including the CMS and ATLAS [1920] constraints as dashed (solid) lines, the latter with a red band indicating the estimated theoretical uncertainty in the calculation of $M_{h}$ of $\sim 1.5 \mathrm{GeV}$, and the pre-LHC $\chi^{2}$ function is shown as a dotted line.

erences for larger $m_{1 / 2}$ and larger $\tan \beta$. We have recalculated the values of $\sigma_{p}^{\mathrm{SI}}$ at the best-fit points in the different models assuming $\Sigma_{\pi N}=45 \mathrm{MeV}$, close to the lower end of the plausible range [57, and found reductions in $\sigma_{p}^{\mathrm{SI}}$ by factors $\sim 3$ to 4 in each case.

Fig. 7 displays the regions of the $\left(m_{\tilde{\chi}_{1}^{0}}, \sigma_{p}^{\mathrm{SI}}\right)$ planes favoured at the $68 \%$ and $95 \%$ CL in the CMSSM, NUHM1, VCMSSM and mSUGRA models, assuming $\Sigma_{\pi N}=64 \mathrm{MeV}$, comparing the post- and pre-LHC contours (dashed/solid lines and dotted lines, respectively), and showing the best-fit points as solid and open stars, respec- 

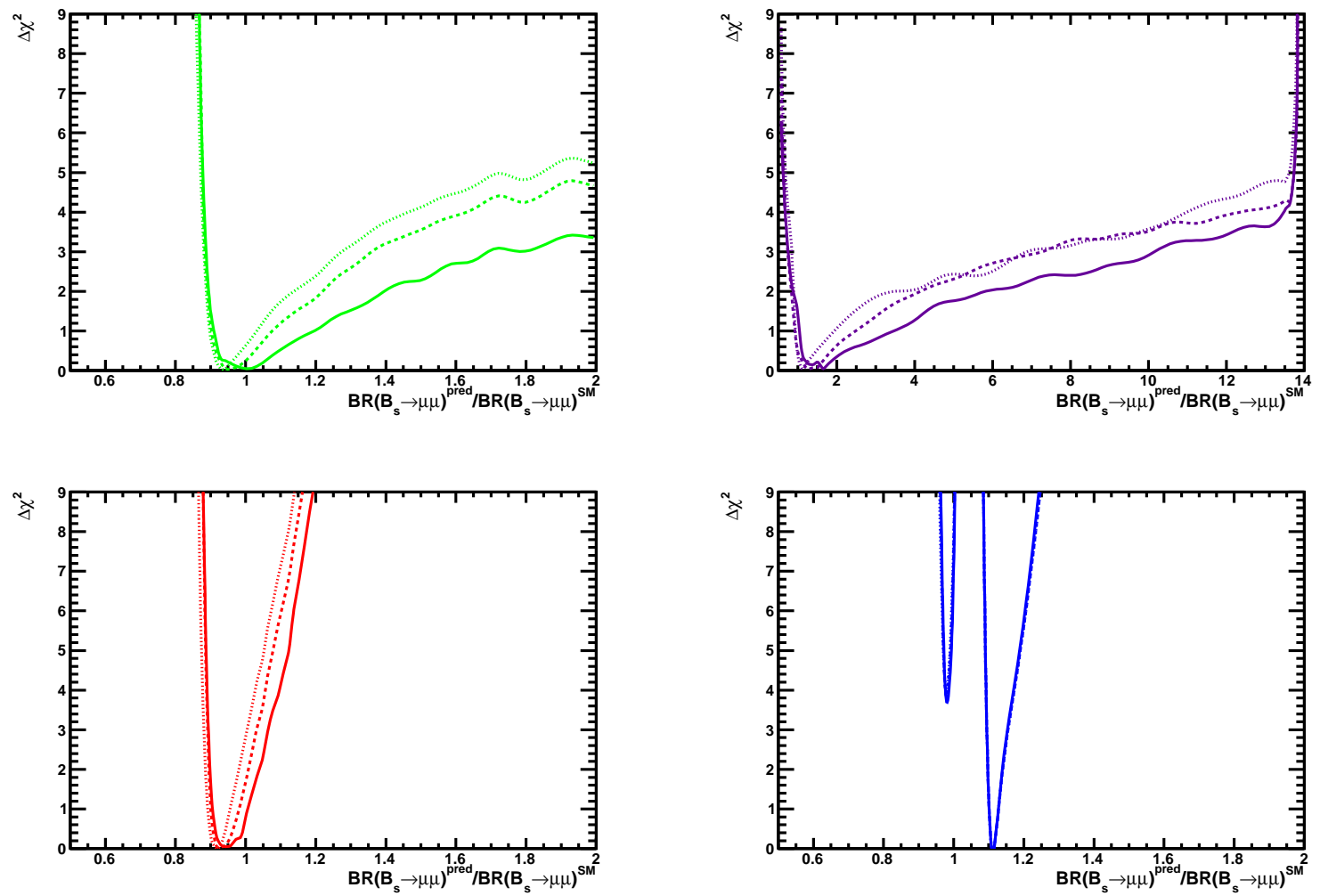

Figure 5. The one-parameter $\chi^{2}$ likelihood functions for $\mathrm{BR}\left(B_{s} \rightarrow \mu^{+} \mu^{-}\right)$in the CMSSM (upper left), NUHM1 (top right), VCMSSM (lower left) and mSUGRA (lower right). In each panel, we show the $\chi^{2}$ function including the CMS and ATLAS [1920] constraints as dashed and solid lines, respectively, and the previous $\chi^{2}$ function as a dotted line.

tively 9. The models predict, in general, a negative correlation between $m_{\tilde{\chi}_{1}^{0}}$ and $\sigma_{p}^{\mathrm{SI}}$, which is why the stronger lower limit on $m_{\tilde{g}}$ and hence $m_{\tilde{\chi}_{1}^{0}}$ provided by CMS and ATLAS (see Fig. 3) corresponds to a stronger upper limit on $\sigma_{p}^{\text {SI }}$, as seen in Fig. 6. Conversely, any future upper limit on (measurement of) $\sigma_{p}^{\text {SI }}$ would correspond to a lower limit on (preferred range of) $m_{\tilde{\chi}_{1}^{0}}$ and hence $m_{\tilde{g}}$. However, this correlation is weakened by the uncertainty in $\Sigma_{\pi N}$, which should be taken into account in the interpretation of any future constraint on $\sigma_{p}^{\mathrm{SI}}$.

\footnotetext{
${ }^{9}$ The region at small $m_{\tilde{\chi}_{1}^{0}}$ in the mSUGRA plot corresponds to the light Higgs funnel discussed in 17.
}

In summary, we have shown that the initial CMS and ATLAS searches for supersymmetry at the LHC at $7 \mathrm{TeV}$ already have significant impacts on the regions of the CMSSM, NUHM1 and VCMSSM parameter spaces favoured at the 68 and $95 \%$ CL, shifting the best-fit points to somewhat larger values (with an estimated systematic uncertainty of $\sim 10 \%$ (CMS) to $20 \%$ (ATLAS) in $m_{1 / 2}$ ). They are now located at $\left(m_{1 / 2}, m_{0}\right) \sim(340$ to 490,100$) \mathrm{GeV}$, corresponding to $m_{\tilde{g}} \sim 800$ to $1000 \mathrm{GeV}$, within the prospective reach of the LHC with $1 / \mathrm{fb}$ of analyzed data at a centre-of-mass energy of $7 \mathrm{TeV}$ or more 10.

${ }^{10}$ However, we recall that significantly larger values of $m_{\tilde{g}}$ are allowed at the $95 \%$ CL. 

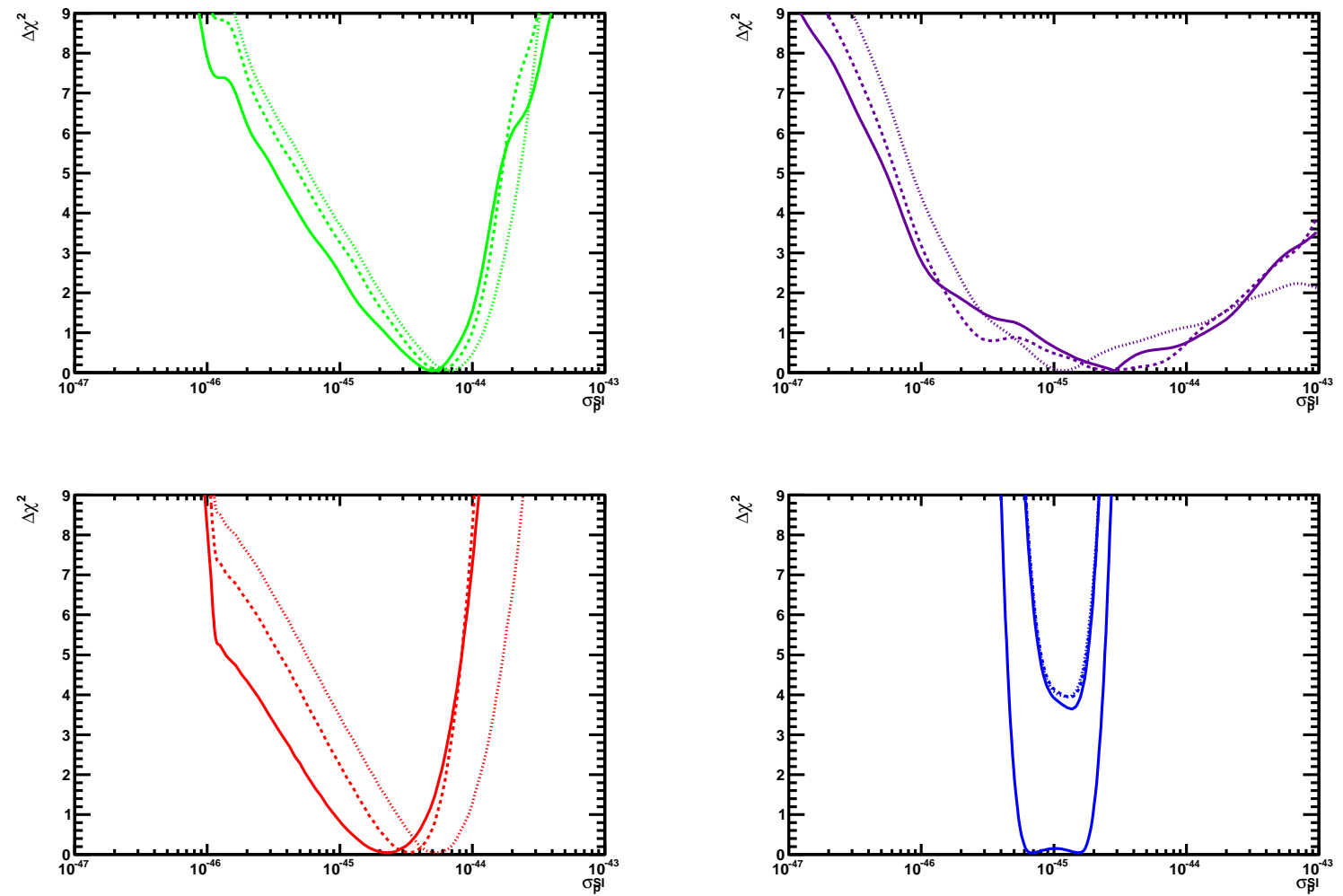

Figure 6. The one-parameter $\chi^{2}$ likelihood functions for the spin-independent neutralino dark matter scattering cross section $\sigma_{p}^{\text {SI }}$ in the CMSSM (upper left), NUHM1 (top right), VCMSSM (lower left) and mSUGRA (lower right). In each panel, we show the $\chi^{2}$ function calculated assuming $\Sigma_{\pi N}=64 \mathrm{MeV}$ and including the CMS and ATLAS [1920] constraints as dashed and solid lines, and the previous $\chi^{2}$ function for $\Sigma_{\pi N}$ as a dotted line.

On the other hand, the CMS and ATLAS data have no impact on the mSUGRA fit. Increases in the preferred values of $\tan \beta$ in the CMSSM and particularly the NUHM1 increase the likelihood that $\operatorname{BR}\left(B_{s} \rightarrow \mu^{+} \mu^{-}\right)$exceeds significantly the SM value. The model predictions for $M_{h}$ (discarding the direct searches by LEP) are now in better agreement with the direct search limits, because of the somewhat heavier mass spectrum required by CMS and ATLAS. Within the NUHM1, the new $\chi^{2}$ contribution decreases significantly the likelihood of a light Higgs boson with $M_{h} \lesssim 110 \mathrm{GeV}$. Within the CMSSM and VCMSSM frameworks, the CMS and ATLAS results also diminish significantly the likelihood that $\sigma_{p}^{\mathrm{SI}}>10^{-44} \mathrm{~cm}^{2}$, but have little impact in the NUHM1 and mSUGRA.

The search for supersymmetry is about to enter its critical stage, and further results from the LHC with tailored analyses and increased luminosity will soon provide crucial new information, as will dark matter search experiments.

\section{Note added}

During the completion of this work three papers addressing related topics appeared [58159. 60. Ref. [58 studies the phenomenology of the mSUGRA rapid-annihilation funnel region. 

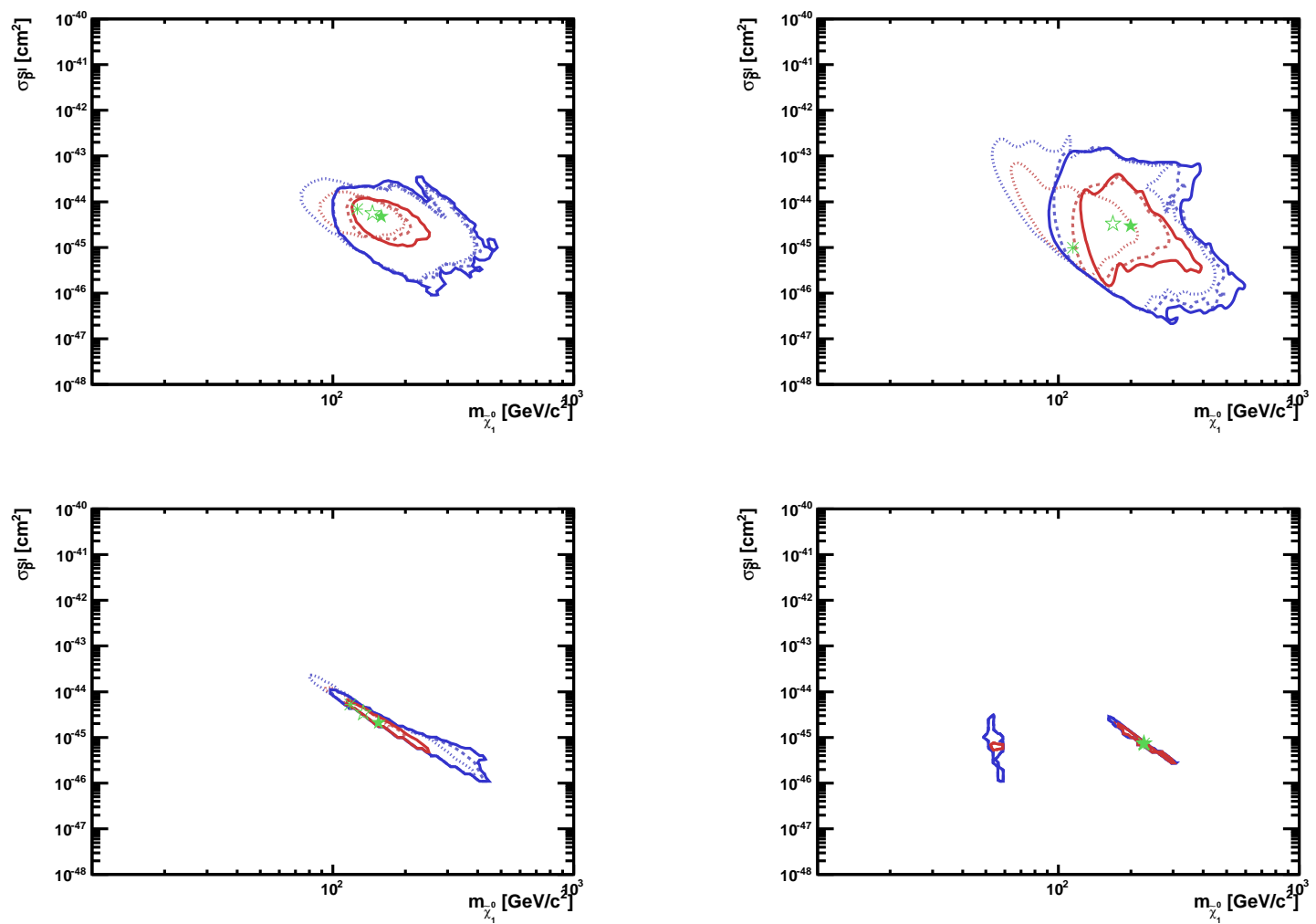

Figure 7. The regions of the $\left(m_{\tilde{\chi}_{1}^{0}}, \sigma_{p}^{S I}\right)$ planes in the CMSSM (upper left), NUHM1 (top right), VCMSSM (lower left) and mSUGRA (lower right) favoured at the 68\% and 95\% CL including the CMS constraint (solid lines) and excluding it (dashed lines). The best-fit points after (before) the CMS and ATLAS constraints [1920] are shown as open (solid) green stars, and the best pre-LHC fits as green 'snowflakes'. The results are calculated assuming $\Sigma_{\pi N}=64 \mathrm{MeV}$.

Ref. [59] analyzes the CMS constraint within the CMSSM and reaches similar conclusions on the increases in $m_{1 / 2}$ and $\tan \beta$ that it entails. Ref. 60] considers a more general model than those examined here.

This work was supported in part by the European Community's Marie-Curie Research Training Network under contracts MRTN-CT-2006035505 'Tools and Precision Calculations for Physics Discoveries at Colliders' and MRTN-CT2006-035482 'FLAVIAnet', and by the Spanish MEC and FEDER under grant FPA2005-01678. The work of S.H. was supported in part by CI-
CYT (grant FPA 2007-66387 and FPA 201022163-C02-01), and the work of K.A.O. was supported in part by DOE grant DE-FG02-94ER40823 at the University of Minnesota. K.A.O. also thanks SLAC (supported by the DOE under contract number DE-AC02-76SF00515) and the Stanford Institute for Theoretical Physics for their hospitality and support while this work was being finished.

\section{REFERENCES}

1. G. Aad et al. ATLAS Collaboration, Expected Performance of the ATLAS Experiment - De- 
tector, Trigger and Physics, arXiv:0901.0512

2. G. L. Bayatian et al., CMS Collaboration, CMS Technical Design Report, Volume II: Physics Performance, CERN-LHCC-2006-021, CMS-TDR008-2 J. Phys. G34, 995 (2007); see: http://cmsdoc.cern.ch/cms/cpt/tdr/.

3. J. Ellis, J.S. Hagelin, D.V. Nanopoulos, K.A. Olive and M. Srednicki, Nucl. Phys. B 238 (1984) 453; H. Goldberg, Phys. Rev. Lett. 50 (1983) 1419.

4. L. Maiani, All You Need To Know About The Higgs Boson, Proceedings of the Gif-surYvette Summer School On Particle Physics, 1979, pp.1-52; G. 't Hooft, in Recent developments in Gauge Theories, Proceedings of the NATO Advanced Study Institute, Cargèse, 1979, eds. G. 't Hooft et al. (Plenum Press, NY, 1980); E. Witten, Phys. Lett. B 105 (1981) 267.

5. The Muon g-2 Collaboration, Phys. Rev. Lett. 92 (2004) 161802. arXiv:hep-ex/0401008; G. Bennett et al. [The Muon g-2 Collaboration], Phys. Rev. D 73 (2006) 072003 arXiv:hep-ex/0602035.

6. D. Stockinger, J. Phys. G 34 (2007) R45 arXiv:hep-ph/0609168; J. Miller, E. de Rafael and B. Roberts, Rept. Prog. Phys. 70 (2007) 795 arXiv:hep-ph/0703049; J. Prades, E. de Rafael and A. Vainshtein, arXiv:0901.0306 [hep-ph]; F. Jegerlehner and A. Nyffeler, Phys. Rept. 477, 1 (2009) arXiv:0902.3360 [hep-ph]]; J. Prades, Acta Phys. Polon. Supp. 3, 75 (2010) arXiv:0909.2546 [hep-ph]]; T. Teubner, K. Hagiwara, R. Liao, A. D. Martin and D. Nomura, arXiv:1001.5401 [hep-ph].

7. M. Davier, A. Hoecker, B. Malaescu and Z. Zhang, arXiv:1010.4180 [hep-ph].

8. J. R. Ellis, G. Ridolfi and F. Zwirner, Phys. Lett. B 257 (1991) 83; Phys. Lett. B 262 (1991) 477; Yasuhiro Okada, Masahiro Yamaguchi and Tsutomu Yanagida, Phys. Lett. B262, 54, 1991; Prog. Theor. Phys. 85, 1, 1991; A. Yamada, Phys. Lett. B 263, 233 (1991); Howard E. Haber and Ralf Hempfling, Phys. Rev. Lett. 66, 1815, 1991; M. Drees and M. M. Nojiri, Phys. Rev. D 45 (1992) 2482;
P. H. Chankowski, S. Pokorski and J. Rosiek, Phys. Lett. B 274 (1992) 191; Phys. Lett. B 286 (1992) 307;

9. G. Degrassi, S. Heinemeyer, W. Hollik, P. Slavich and G. Weiglein, Eur. Phys. J. C 28 (2003) 133 arXiv:hep-ph/0212020.

10. W. de Boer and C. Sander, Phys. Lett. B 585 (2004) 276 arXiv:hep-ph/0307049; G. Belanger, F. Boudjema, A. Cottrant, A. Pukhov and A. Semenov, Nucl. Phys. B 706 (2005) 411 arXiv:hep-ph/0407218; J. R. Ellis, K. A. Olive, Y. Santoso and V. C. Spanos, Phys. Rev. D 69 (2004) 095004 arXiv:hep-ph/0310356; J. R. Ellis, S. Heinemeyer, K. A. Olive and G. Weiglein, JHEP 0502 (2005) 013 arXiv:hep-ph/0411216; J. R. Ellis, D. V. Nanopoulos, K. A. Olive and Y. Santoso, Phys. Lett. B 633 (2006) 583 arXiv:hep-ph/0509331; J. R. Ellis, S. Heinemeyer, K. A. Olive and G. Weiglein, JHEP 0605 (2006) 005 arXiv:hep-ph/0602220; J. Ellis, S. Heinemeyer, K.A. Olive, A.M. Weber, G. Weiglein, JHEP 08 (2007) 083 arXiv:0706.0652 [hep-ph]]; J. Ellis, T. Hahn, S. Heinemeyer, K. A. Olive and G. Weiglein, JHEP 0710 (2007) 092 arXiv:0709.0098 [hep-ph]]; J. R. Ellis, S. Heinemeyer, K. A. Olive and G. Weiglein, Phys. Lett. B 653 (2007) 292 arXiv:0706.0977 [hep-ph]]; S. Heinemeyer, X. Miao, S. Su and G. Weiglein, JHEP 0808 (2008) 087 arXiv:0805.2359 [hep-ph]]; P. Bechtle, K. Desch and P. Wienemann, Comput. Phys. Commun. 174 (2006) 47 arXiv:hep-ph/0412012; R. Lafaye, T. Plehn, M. Rauch and D. Zerwas, Eur. Phys. J. C 54 (2008) 617 arXiv:0709.3985 [hep-ph]]; E. A. Baltz and P. Gondolo, JHEP 0410 (2004) 052 arXiv:hep-ph/0407039; B. C. Allanach and C. G. Lester, Phys. Rev. D 73 (2006) 015013 arXiv:hep-ph/0507283; B. C. Allanach, Phys. Lett. B 635 (2006) 123 arXiv:hep-ph/0601089; B. C. Allanach, C. G. Lester and A. M. Weber, JHEP 0612 (2006) 065 arXiv:hep-ph/0609295; B. C. Allanach and C. G. Lester, Comput. Phys. Commun. 179 (2008) 256 arXiv:0705.0486 [hep-ph]]; B. C. Allanach, K. Cranmer, 
C. G. Lester and A. M. Weber, JHEP 0708, 023 (2007) arXiv:0705.0487 [hep-ph]]; B. C. Allanach and D. Hooper, JHEP 0810 (2008) 071 arXiv:0806.1923 [hepph]]; F. Feroz, B. C. Allanach, M. Hobson, S. S. AbdusSalam, R. Trotta and A. M. Weber, JHEP 0810 (2008) 064 arXiv:0807.4512 [hep-ph]]; R. R. de Austri, R. Trotta and L. Roszkowski, JHEP 0605 (2006) 002 arXiv:hep-ph/0602028; L. Roszkowski, R. R. de Austri and R. Trotta, JHEP 0704 (2007) 084 arXiv:hep-ph/0611173; L. Roszkowski, R. Ruiz de Austri and R. Trotta, JHEP $\mathbf{0 7 0 7}$ (2007) 075 arXiv:0705.2012 [hep-ph]]; L. Roszkowski, R. R. de Austri, J. Silk and R. Trotta, Phys. Lett. B 671 (2009) 10 arXiv:0707.0622 [astro-ph]]; R. Trotta, F. Feroz, M. P. Hobson, L. Roszkowski and R. Ruiz de Austri, JHEP 0812 (2008) 024 arXiv:0809.3792 [hep-ph]]; S. S. AbdusSalam, B. C. Allanach, M. J. Dolan, F. Feroz and M. P. Hobson, Phys. Rev. D $\mathbf{8 0} \quad$ (2009) 035017 arXiv:0906.0957 [hep-ph]]; G. Belanger, F. Boudjema, A. Pukhov and R. K. Singh, JHEP 0911 (2009) 026 arXiv:0906.5048 [hep-ph]]; S. S. AbdusSalam, B. C. Allanach, F. Quevedo, F. Feroz and M. Hobson, Phys. Rev. D 81 (2010) 095012 arXiv:0904.2548 [hep-ph]]; P. Bechtle, K. Desch, M. Uhlenbrock and P. Wienemann, Eur. Phys. J. C 66 (2010) 215 arXiv:0907.2589 [hep-ph]]; M. Cabrera, A. Casas and R. Ruiz de Austri, JHEP 1005 (2010) 043 arXiv:0911.4686 [hep-ph]]; M. Bridges, K. Cranmer, F. Feroz, M. Hobson, R. R. de Austri and R. Trotta, arXiv:1011.4306 [hep-ph]; L. Roszkowski, R. Ruiz de Austri and R. Trotta, Phys. Rev. D 82 (2010) 055003 arXiv:0907.0594 [hep-ph]];

11. M. Drees and M. M. Nojiri, Phys. Rev. D 47 (1993) 376 arXiv:hep-ph/9207234]; H. Baer and M. Brhlik, Phys. Rev. D 53 (1996) 597 arXiv:hep-ph/9508321; Phys. Rev. D 57 (1998) 567 arXiv:hep-ph/9706509; J. R. Ellis, T. Falk, K. A. Olive and M. Schmitt, Phys. Lett. B 388 (1996) 97 arXiv:hep-ph/9607292; Phys. Lett. B
413 (1997) 355 arXiv:hep-ph/9705444; J. R. Ellis, T. Falk, G. Ganis, K. A. Olive and M. Schmitt, Phys. Rev. D 58 (1998) 095002 arXiv:hep-ph/9801445; V. D. Barger and C. Kao, Phys. Rev. D 57 (1998) 3131 arXiv:hep-ph/9704403; J. R. Ellis, T. Falk, G. Ganis and K. A. Olive, Phys. Rev. D 62 (2000) 075010 arXiv:hep-ph/0004169; J. R. Ellis, T. Falk, G. Ganis, K. A. Olive and M. Srednicki, Phys. Lett. B 510 (2001) 236 arXiv:hep-ph/0102098; V. D. Barger and C. Kao, Phys. Lett. B 518 (2001) 117 arXiv:hep-ph/0106189; $\quad$ L. Roszkowski, R. Ruiz de Austri and T. Nihei, JHEP 0108 (2001) 024 arXiv:hep-ph/0106334; A. Djouadi, M. Drees and J. L. Kneur, JHEP 0108 (2001) 055 arXiv:hep-ph/0107316; U. Chattopadhyay, A. Corsetti and P. Nath, Phys. Rev. D 66 (2002) 035003 arXiv:hep-ph/0201001; $\quad$ J. R. Ellis, K. A. Olive and Y. Santoso, New Jour. Phys. 4 (2002) 32 arXiv:hep-ph/0202110; H. Baer, C. Balazs, A. Belyaev, J. K. Mizukoshi, X. Tata and Y. Wang, JHEP 0207 (2002) 050 arXiv:hep-ph/0205325; R. Arnowitt and B. Dutta, arXiv:hep-ph/0211417.

12. O. Buchmueller et al., Phys. Lett. B 657 (2007) 87 arXiv:0707.3447 [hep-ph]].

13. H. Baer, A. Mustafayev, S. Profumo, A. Belyaev and X. Tata, Phys. Rev. D 71, 095008 (2005) arXiv:hep-ph/0412059; H. Baer, A. Mustafayev, S. Profumo, A. Belyaev and X. Tata, JHEP 0507 (2005) 065, hep-ph/0504001; J. R. Ellis, K. A. Olive and P. Sandick, Phys. Rev. D 78, 075012 (2008) arXiv:0805.2343 [hep-ph]].

14. O. Buchmueller et al., JHEP 0809 (2008) 117 arXiv:0808.4128 [hep-ph]].

15. O. Buchmueller et al., Eur. Phys. J. C 64, 391 (2009) arXiv:0907.5568 [hep-ph]].

16. J. R. Ellis, K. A. Olive, Y. Santoso and V. C. Spanos, Phys. Lett. B 573 (2003) 162 arXiv:hep-ph/0305212, and Phys. Rev. D 70 (2004) 055005 arXiv:hep-ph/0405110].

17. O. Buchmueller et al., arXiv:1011.6118 [hep$\mathrm{ph}$.

18. See: http://cern.ch/mastercode.

19. V. Khachatryan et al., CMS Collaboration, 
arXiv:1101.1628 [hep-ex].

20. G. Aad et al. ATLAS Collaboration, arXiv:1102.2357 [hep-ex].

21. For reviews, see: H. P. Nilles, Phys. Rep. 110 (1984) 1; A. Brignole, L. E. Ibanez and C. Munoz, arXiv:hep-ph/9707209, published in Perspectives on supersymmetry, ed. G. L. Kane, pp. 125-148; R. Barbieri, S. Ferrara and C. A. Savoy, Phys. Lett. B 119, 343 (1982).

22. O. Buchmueller et al., Phys. Rev. D 81 (2010) 035009 arXiv:0912.1036 [hep-ph]].

23. Tevatron Electroweak Working Group and CDF Collaboration and DØCollaboration, arXiv:1007.3178 [hep-ex].

24. B. Bhattacherjee et al., arXiv:1012.1052 [hep$\mathrm{ph}$.

25. M. Bona et al. [UTfit Collaboration], Phys. Lett. B 687 (2010) 61 arXiv:0908.3470 [hep$\mathrm{ph}]]$.

26. M. Antonelli et al., Eur. Phys. J. C 69, 399 (2010) arXiv:1005.2323 [hep-ph]].

27. B. C. Allanach, Comput. Phys. Commun. 143 (2002) 305 arXiv:hep-ph/0104145.

28. S. Heinemeyer, W. Hollik and G. Weiglein, Eur. Phys. J. C 9 (1999) 343 arXiv:hep-ph/9812472.

29. S. Heinemeyer, W. Hollik and G. Weiglein, Comput. Phys. Commun. 124 (2000) 76 arXiv:hep-ph/9812320. See http://www.feynhiggs.de .

30. M. Frank, T. Hahn, S. Heinemeyer, W. Hollik, H. Rzehak and G. Weiglein, JHEP 0702 (2007) 047 arXiv:hep-ph/0611326.

31. G. Isidori and P. Paradisi, Phys. Lett. B 639 (2006) 499 arXiv:hep-ph/0605012.

32. G. Isidori, F. Mescia, P. Paradisi and D. Temes, Phys. Rev. D 75 (2007) 115019 arXiv:hep-ph/0703035, and references therein.

33. F. Mahmoudi, Comput. Phys. Commun. 178 (2008) 745 arXiv:0710.2067 [hep-ph]] and arXiv:0808.3144 [hep-ph].

34. D. Eriksson, F. Mahmoudi and O. Stal, JHEP 0811 (2008) 035 arXiv:0808.3551 [hep-ph]].

35. S. Heinemeyer, W. Hollik, D. Stockinger, A. M. Weber and G. Weiglein, JHEP 0608 (2006) 052 arXiv:hep-ph/0604147.
36. S. Heinemeyer, W. Hollik, A. M. Weber and G. Weiglein, JHEP 0804 (2008) 039 arXiv:0710.2972 [hep-ph]].

37. G. Belanger, F. Boudjema, A. Pukhov and A. Semenov, Comput. Phys. Commun. 176 (2007) 367 arXiv:hep-ph/0607059.

38. G. Belanger, F. Boudjema, A. Pukhov and A. Semenov, Comput. Phys. Commun. 149 (2002) 103 arXiv:hep-ph/0112278.

39. G. Belanger, F. Boudjema, A. Pukhov and A. Semenov, Comput. Phys. Commun. 174 (2006) 577 arXiv:hep-ph/0405253.

40. P. Gondolo, J. Edsjo, P. Ullio, L. Bergstrom, M. Schelke and E. A. Baltz, New Astron. Rev. 49 (2005) 149.

41. P. Gondolo, J. Edsjo, P. Ullio, L. Bergstrom, M. Schelke and E. A. Baltz, JCAP 0407 (2004) 008 arXiv:astro-ph/0406204.

42. P. Skands et al., JHEP 0407 (2004) 036 arXiv:hep-ph/0311123.

43. B. Allanach et al., Comput. Phys. Commun. 180 (2009) 8 [arXiv:0801.0045 [hep-ph]].

44. G. Aad et al., ATLAS Collaboration, http://cdsweb.cern.ch/record/1323868/ files/ATL-PHYS-PUB-2011-003.pdf.

45. G. Aad et al., ATLAS Collaboration, http://cdsweb.cern.ch/record/1278474/ files/ATL-PHYS-PUB-2010-010.pdf.

46. R. Barate et al. [ALEPH, DELPHI, L3, OPAL Collaborations and LEP Working Group for Higgs boson searches], Phys. Lett. B 565 (2003) 61 arXiv:hep-ex/0306033.

47. S. Schael et al. [ALEPH, DELPHI, L3, OPAL Collaborations and LEP Working Group for Higgs boson searches], Eur. Phys. J. C $\mathbf{4 7}$ (2006) 547 arXiv:hep-ex/0602042.

48. Y. Akrami, C. Savage, P. Scott, J. Conrad and J. Edsjo, arXiv:1011.4297 [hep-ph] and M. Bridges, K. Cranmer, F. Feroz, M. Hobson, R. R. de Austri and R. Trotta, in [10.

49. CDF and D $\varnothing$ Collaborations, arXiv:1007.4587 [hep-ex].

50. G. Aad et al. ATLAS Collaboration, ATLASCONF-2011-005.

51. J. R. Ellis, S. Heinemeyer, K. A. Olive and G. Weiglein, Phys. Lett. B 515 (2001) 348 arXiv:hep-ph/0105061.

52. S. Ambrosanio, A. Dedes, S. Heinemeyer, 
S. Su and G. Weiglein, Nucl. Phys. B 624 (2002) 3 arXiv:hep-ph/0106255.

53. J. R. Ellis, K. Enqvist, D. V. Nanopoulos and F. Zwirner, Mod. Phys. Lett. A 1, 57 (1986); see also R. Barbieri and G. F. Giudice, Nucl. Phys. B 306 (1988) 63.

54. A. Strumia, arXiv:1101.2195 [hep-ph].

55. B. Adeva et al. [The LHCb Collaboration], Roadmap for selected key measurements of $L H C b$, arXiv:0912.4179 [hep-ex].

56. E. Aprile et al. [XENON100 Collaboration], Phys. Rev. Lett. 105 (2010) 131302 arXiv:1005.0380 [astro-ph.CO]].

57. J. R. Ellis, K. A. Olive and C. Savage, Phys. Rev. D 77 (2008) 065026 arXiv:0801.3656 [hep-ph]].

58. D. Feldman, K. Freese, P. Nath, B. D. Nelson and G. Peim, arXiv:1102.2548 [hep-ph].

59. B. C. Allanach, arXiv:1102.3149 [hep-ph].

60. S. Scopel, S. Choi, N. Fornengo and A. Bottino, arXiv:1102.4033 [hep-ph]. 